Recebido em 16/07/2018. Aprovado em 13/09/2018. Avaliado pelo sistema cabdeblindpeerreviex Publicado conforme normas da ABNT. http://dx.doi.org/10.22279/navus.2019.v9n3.p55-68.819

PechoVeiraSaraSantos Engenheiro de Produção. Universidade Federal do Vale do São Francisco (UNIVASF) - Brasil. pedroveirass@hotmail.com

\title{
A introdução de tecnologias a favor da eficiência em operações logísticas: um estudo de caso no setor de serviços
}

RESUMO

O uso cada vez maior de tecnologias em ambientes empresariais, como suporte às operações, é notório. Ademais, o uso de ferramentas tecnológicas, em especial na logística e sua gestão, é considerada uma das formas mais eficientes de melhorar os negócios. Assim, para que as atividades logísticas possam ser executadas, o uso de tecnologias pode favorecer o objetivo primordial dessa área. Em face desse cenário, o intuito do presente trabalho foi definido como analisar o impacto da introdução de tecnologias em operações logísticas em uma empresa de serviços localizada em Pernambuco, comparando a evolução do setor antes e depois da utilização do aparato tecnológico em seu cotidiano. Como resultado, é notório que o programa cumpriu com seu objetivo de melhorar as operações do setor logístico da empresa, trazendo uma abordagem mais realista do que acontece no cotidiano do departamento, facilitando ainda a tomada de decisão por parte da gestão local.

Palavras-chave: Tecnologia. Gestão. Logística.

\section{Introduction of technologies in favor of efficiency in logistics: a case study in the service sector}

\begin{abstract}
The increasing use of technologies in business environments, such as support to operations, is notorious. In addition, the use of technological tools, especially in logistics and its management, is considered one of the most efficient ways of improving the business. Thus, in order for logistic activities to be carried out, the use of technologies may favor the primary objective of this area. In view of this scenario, the purpose of this paper was to analyze the impact of the introduction of technologies in logistic operations in a service company located in Pernambuco, comparing the evolution of the sector prior and after the use of the technological apparatus in its routines. As a result, it is clear that the program has fulfilled its objective of improving the operations of the logistics sector of the company, bringing a more realistic approach to the day by day routines of the department, and also facilitating decision making process by local management.
\end{abstract}

Keywords: Technology. Management. Logistics. 


\section{INTRODUÇÃO}

Diante de um ambiente de negócios cada vez mais acirrado, as organizações devem desenvolver competências capazes de mantê-las competitivas. Nesse sentido, fatores como a velocidade na tomada de decisão, capacidade de adaptação a mudanças, resposta adequada às demandas dos clientes e inovação, são exemplos que atendem ao esforço que as empresas podem fazer para acompanhar a instabilidade do ambiente mercadológico (MAŠlĆ; ; DŽUNIĆ; NEŠIĆ, 2014; SANTOS; ROCHA, 2017). Portanto, Reeves e Deimler (2011) afirmam que o posicionamento da empresa diante das exigências que surgem constantemente é uma das competências a serem ap rimoradas.

Nesse cenário, a gestão bem executada é de fundamental importância na garantia da qualidade das operações da empresa, de forma geral. Potocan, Nedelko e Mulej (2012) citam que na literatura pode-se encontrar diferentes abordagens acerca do termo gestão. Para os autores, há diversos estágios que fomentam o conceito da palavra, como metodologias, métodos, técnicas e ferramentas, todas associadas a implementação de ações gerenciais. Segundo Muniz Junior (2012, p. 18-20) o ato de gerir tem o “objetivo de organizar a forma com que as empresas geram os produtos e serviços utilizando da melhor forma seus recursos disponíveis (homens, máquinas, tecnologia etc)."

De acordo com Araújo (2004, p. 110-111), uma empresa "é estruturada por diversas áreas que, juntas, ditam sua vida." Assim, pode-se compreender que a organização deve, essencialmente, garantir um bom funcionamento e integração entre os setores internos, sob comando da gestão local; uma destas é a logística.

Para Bowersox e Closs (2001, p. 19) o termo logística pode ser entendido como um processo “[...] de planejamento, implementação e controle eficiente e eficaz do fluxo e armazenagem de mercadorias, serviços e informações relacionadas, desde o ponto de origem até o ponto de consumo, com o objetivo de atender as necessidades dos clientes." Kherbach e Mocan (2016) reportam que, devido ao processo de desenvolvimento da economia, assim como da globalização, a logística é uma ferramenta importante nesse processo de atualização dos mercados.

Na visão de Li (2014), dos recursos nos quais são gerenciados pela logística, podem ser considerados itens físicos, como alimentos, materiais, animais, equipamentos e líquidos, bem como itens abstratos, como tempo, informação e energia. Envolve ainda o fluxo e transporte de produtos, do armazém e/ou depósito até o consumidor.

Nesse contexto, para que as atividades logísticas possam ser executadas, o uso de tecnologias pode favorecer o objetivo primordial dessa área, isto é, entregar a quantidade certa, em local e tempo certo (CHRISTOPHER, 1992). Ballou (2007) complementa ao afirmar que a logística é um setor com constantes oportunidades de aperfeiçoamento dos seus processos e/ou atividades.

Bowersox, Closs e Cooper (2007) citam que a tecnologia é capaz de ap rimorar diversas ações do ramo logístico, desde o pedido ao relacionamento entre os elos da cadeia de suprimentos. Por Tecnologia pode-se compreender como uma infraestrutura que processa as informações que as empresas possuem, criam e utilizam (LAURINDO, 2008). Logo, o desenvolvimento e aplicação de aparato tecnológico em setores como a logística, proporcionam, sob o enfoque da gestão, a integração de informações, sendo capaz ainda de coordenar as práticas operacionais e priorizar a eficiência (YUCESAN, 2007).

A tecnologia possui então a característica de ser um forte pilar da administração integrada na logística (VIJ AYASARATHY, 2010). Ademais, o uso de ferramentas tecnológicas, em especial na logística e sua gestão, é considerada uma das formas mais eficientes de melhorar os negócios (LAKATOS; NÉMETH, 2013).

Portanto, isto posto, o objetivo do presente trabalho é analisar o impacto da introdução de tecnologias em operações logísticas em uma empresa de serviços localizada em Pernambuco, comparando a evolução do setor antes e depois da utilização do aparato tecnológico em seu cotidiano. 


\section{ABORDAGEM TEÓRICA}

O ambiente ágil e competitivo hoje requer das organizações empresariais maior atenção quanto 0 modo de trabalhar, ou seja, sua gestão de forma geral (GUPTA; RAMESH 2015). Isto posto, as organizações são induzidas a direcionarem seus esforços em uma administração com foco na eficiência, coordenados para atingir os objetivos estabelecidos pela organização (TATARYNOWICZ; SYTCH; GULATI, 2016). A gestão, como função, deve trabalhar para identificar necessidades locais que permitam a empresa evoluir, atendendo a demandas e criando qualidade em forma de melhoria contínua (GHOUMRASSI; TIGU, 2017).

\subsection{Gestão empresarial}

$\mathrm{Na}$ literatura é possível notar uma gama de conceitos inerentes ao termo gestão ou administração empresarial. Para Daft (2005) a palavra gestão pode ser tratada como ação de atingir metas definidas para a organização, sendo este curso eficaz por meio de planejamento, organização, liderança e controle de recursos organizacionais. Nesse sentido, Certo $(2003$, p. 3) afirma que a gestão pode ser entendida como uma "série de atividades contínuas e relacionadas, implica alcançar os objetivos da empresa e se concentra nisso, alcança esses objetivos fazendo uso do trabalho com e por meio de pessoas e outros recursos da empresa".

Na visão de Andrade e Amboni (2007) o termo está ligado ao desempenho da organização de forma geral, diante de um determinado contexto. Quetti, Pigni e Clerici (2012), completam ao afirmarem que os modelos de gestão desenvolvidos atualmente devem atualizar-se de acordo com as mudanças que ocorrem em meio ao ambiente mercadológico, caracterizando-se pelo alto grau de competitividade. Maximiano (2000, p. 265) afirma que a gestão organizacional pode ser traduzida como "[...] o processo de organizar, ou processo de organização, cria uma gestão estável e dinâmica, que define o trabalho que as pessoas, como indivíduos ou integrantes de grupos, devem realizar.

Bonazzi e Zilber (2014), comentam que há uma notória necessidade de buscar criar valor como parte de uma visão estratégica da administração, para que o modelo de negócio adotado pela empresa esteja em destaque. Eschenbächer, Seifert e Thoben (2011) acrescentam que esta capacidade de inovar por parte das organizações é essencial para que as mesmas permaneçam competitivas no mercado.

Govindaraja e Natarajan (2005) sintetizam a definição do termo gestão ou gerência como uma arte e ciência que engloba todas as atividades dentro de uma organização, sendo o núcleo de cada instituição, organização, empresa. Tal empreendimento pode ser micro, macro, público ou privado, em todos há necessidade dessa administração, que envolve o planejamento, coordenação, controle, organização de todas as atividades que acontecem em seu ambiente interno.

A gestão traduz-se, em suma, como a formulação estratégica de implementação de um plano de gerenciamento dos processos de negócio. Os gestores têm, em seu favor, a possibilidade de empregar uma combinação de diferentes estratégias para atingir os objetivos organizacionais. Para tanto, pode-se observar que este comportamento envolve a análise do produto, cliente, valores, localização, concorrentes, habilidades e recursos disponíveis, oportunidades e riscos, potencial para crescer e outros (MÜNCHHAUSEN; HÄRING, 2016). Nesse sentido, a logística tem papel fundamental.

\subsection{Logística}

Do ponto de vista histórico, a palavra logística é oriunda do termo "logistician", utilizada no tempo de Napoleão Bonaparte para encontro de alojamentos para tropas (VAN CREVELD, 2004). 0 termo logo evoluiu como uma atividade, traduzindo-se como responsável por fluxo de materiais entre pontos distintos.

0 conceito do termo logística agrega diversos elementos e torna-se mais amplo. De acordo com Ballou (2007), trata-se de uma área que envolve os fluxos de material, produtos e informação, abrangendo 
assim os elos entre diferentes empresas da cadeia de suprimentos. Yayla et al. (2015) ainda afirma que as operações logísticas são capazes de garantir a competitividade da cadeia na qual a empresa atua.

Em outras palavras, Novaes (2001, p. 36) conceitua logística como:

O processo de planejar, implementar e controlar de maneira eficiente o fluxo e a armazenagem de produtos, bem como os serviços e informações associados, cobrindo desde 0 ponto de origem até o ponto de consumo, com o objetivo de atender aos requisitos do consumidor.

Ademais, os "sete certos" é uma definição bem consolidada de logística, dada por Christopher (1992), na qual retrata que significa assegurar a disponibilidade dos bens certos, na quantidade certa, em condição certa, no lugar certo, no momento certo para a cliente certo, com os custos certos. Esta definição reflete as exigências nas quais as empresas devem estar atentas, paralelo ao fato de que a complexidade das operações é crescente (ELMARAGHY et al., 2013).

Chankov, Becker e Windt (2014), comenta que, a partir de uma perspectiva ativa, as definições de logística requerem um alinhamento, efetivo, entre atividades, o que implica em uma necessidade notória de sincronização como pré-requisito para que as operações logísticas sejam bem-sucedidas.

A logística atua como uma ferramenta muito importante para integrar o conjunto de operações de uma organização, sendo ainda responsável por garantir a satisfação de seus clientes (GHOUMRASSI; ȚIGU, 2017). Outra consideração importante é que esta área deve ser eficiente e flexível, sendo um requisito para atendimento ao objetivo de ter vantagem competitiva significativa (BEŠLIĆ; BEŠLIĆ, 2010), especialmente em termos de custo e tempo que pertencem aos fatores mais importantes para a competitividade.

Para tanto, para a disseminação das informações em fluxo eficiente, diversas tecnologias dispostas no mercado são capazes de atender a esta demanda por maior desempenho nas atividades do setor, onde, segundo Osipov, Kurths e Zhou (2007), podem formar um regime de operação comum (KORECKÝ, 2012).

\subsection{Tecnologias}

Conforme posto anteriormente, a rede de operações logística pode contar com um componente relevante: a tecnologia. Para Cheng (2011) esse apoio acarreta em coordenação mais efetiva dos processos internos das organizações. De acordo com Bowersox, Closs e Cooper (2007, p. 03), o aparato tecnológico:

[...] vem impulsionando a área de pedido, aumentando a conectividade entre empresas, ao mesmo tempo em que impulsiona gestão da cadeia de suprimentos, que vem a ser uma nova ordem de relacionamentos que permite aos produtos serem fabricados de acordo com especificações exatas e entregues rapidamente a consumidores espalhados pelo mundo todo.

Na ótica de Rezende e Abreu (2011) a tecnologia, com foco na informação, pode ser entendida como recursos computacionais direcionados para a geração da informação. Cruz (2007, p. 186) complementa ainda, ao afirmar que 0 assunto se refere a um "conjunto de dispositivos individuais, como hardware, software, telecomunicações ou qualquer outra tecnologia que, faça parte ou gere tratamento da informação, ou ainda, que a contenha". Em suma, pode-se notar que estes sistemas atuam para obtenção de um melhor fluxo das informações entre departamentos da empresa.

Melville (2010) aponta que, além dos benefícios causados pelo uso da tecnologia em organizações, a mesma ainda contribui para a vantagem competitiva. Além disso, Vijayasarathy (2010) acrescenta que 0 desenvolvimento tecnológico possibilita que a gestão logística se torne um processo coordenado, integrando várias atividades de cunho operacional com foco na eficiência. So e Sun (2010) ainda comentam que esta integração é uma base para que haja um estabelecimento de valor na rede de operações, ou seja, dentre as consequências disto está a maior colaboração entre os agentes.

Para Moura (2010) a tecnologia é uma força que impulsiona a busca por melhorias, principalmente no que diz respeito à lucratividade no campo logístico. Para o autor, a mesma ainda fornece informações no 
tempo certo para apoio a decisões mais acertadas. Bowersox e Closs (2011) comentam que a tecnologia disposta está sendo empregada pelos executivos para elaborar soluções logísticas únicas e inovadoras.

\section{ASPECTOS METODOLÓGICOS}

A seguir são apresentadas a caracterização da pesquisa e suas etapas.

\subsection{Caracterização da pesquisa}

Em relação a metodologia deste trabalho, a mesma apresenta-se como uma pesquisa do tipo exploratória, que segundo Gil $(2009$, p. 41) tem a finalidade de "proporcionar maior familiaridade com 0 problema, com vistas a torná-lo mais explícito ou a constituir hipóteses". Por outro lado, a abordagem do estudo comporta-se como um estudo de caso, que para Yin (2005), é indicado para investigar um conjunto de eventos contemporâneos, dentro de um contexto real.

A pesquisa aqui apresentada pode ser classificada ainda como aplicada, tendo em vista o intuito de gerar conhecimento para intervenção prática na solução de problemas de caráter específicos (SILVA; MENEZES, 2005). Barros e Lehfeld (2000, p. 78), acrescentam que a pesquisa desse tipo motiva-se pela necessidade de produzir conhecimento para aplicação de seus resultados, com o objetivo de "contribuir para fins práticos, visando à solução mais ou menos imediata do problema encontrado na realidade". Appolinário (2004, p. 152) salienta ainda que esse tipo de intervenção busca "resolver problemas ou necessidades concretas e imediatas."

A empresa na qual o estudo foi feito atua no ramo de distribuição de alimentos, localizada na cidade de Petrolina-PE. Como trata-se de uma empresa de origem familiar, diversas necessidades existem, do ponto de vista de gestão, entre elas investimento em tecnologia. Com a adoção de um software de monitoramento da logística de distribuição local, a empresa pôde observar os reais ganhos com tal ferramenta, apresentados e discutidos na sessão de resultados.

\subsection{Das etapas da pesquisa}

Como toda pesquisa de caráter científico, esta seguiu etapas definidas previamente. Com base no objetivo proposto, fez-se uma comparação do estado anterior (sem utilização de tecnologia nas operações do setor) com a situação atual (pós implementação da tecnologia no setor). Para isto, foram coletados dados de relatórios gerenciais da empresa para identificar os valores históricos dos indicadores locais; índices estes, selecionados com base na relevância para a realidade atual do setor.

Foram determinados para submissão as análises deste estudo, indicadores que para a empresa são mais relevantes, sendo: consumo médio de combustível por equipe e a despesa com combustível, devolução mensal de mercadorias, tempo de jornada de trabalho e gasto com hora extra.

Assim, a pesquisa foi realizada entre os meses de janeiro e junho de 2018. Esta pesquisa deu-se então pela sequência das seguintes atividades:

- Reunião inicial para apresentação da proposta: nesta primeira atividade, pôde-se discutir junto aos gestores da empresa, a possiblidade de estudar os benefícios causados pela tecnologia empregada no setor de logística. Isto é, a ideia geral do estudo foi apresentada para consequente aceite da administração local e assim, autorizar que o estudo fosse feito;

- Definição dos objetivos da pesquisa: nesta etapa, foram estabelecidos os objetivos do estudo, alinhados com o propósito da gestão local (que é a busca por melhoria contínua das operações da organização). Logo, firmou-se o propósito central de evidenciar os benefícios trazidos pelo aparato tecnológico para com as operações da empresa. Assim, o setor de logística de distribuição foi escolhido para o procedimento, tendo em vista a importância deste para a empresa e ainda por ser um setor que desempenha atividades-chave e/ou principais no âmbito organizacional; 
- Coleta de dados: A coleta dos dados para a pesquisa, se deu por meio de relatórios gerenciais obtidos pelo sistema eletrônico da empresa. Todos os registros, acerca das operações do setor, são armazenados em um sistema eletrônico de planilhas que permite fácil consulta e disposição para obtenção. A partir deste, teve-se a oportunidade de observar 0 comportamento e/ou resultado dos indicadores (citados anteriormente) do setor de logística de distribuição antes (primeiro trimestre 2018) e depois da aplicação da tecnologia (segundo trimestre 2018);

- Observação dos resultados: Com base nos dados registrados e obtidos na etapa anterior, pôde-se elaborar cenários comparativos, entre os meses em que não foi utilizada tecnologia a favor das operações logística e também após a implementação da mesma no cotidiano da empresa. Foi-se, portanto, posto em comparação os resultad os obtidos no primeiro trimestre e no segundo trimestre de 2018, evidenciando o comportamento dos indicadores ao longo do tempo;

- Entrevista: ao final das observações sobre o programa computacional instalado na empresa para melhorar as operações logísticas, os funcionários do setor foram convocados para participarem, voluntariamente, de um questionário sobre a percepção deles acerca do uso do software. Com isso, pôde-se reforçar a importância e relevância da introdução do aparato tecnológico, especificamente, no setor de logística de distribuição.

\section{RESULTADOS E DISCUSSÃO}

A empresa em questão atua no mercado desde 2005. Contudo, apesar do tempo de participação no mercado, a mesma ainda carece de muito investimento em gestão e tecnologias. Nesse sentido, a direção da organização, com vistas a esta realidade, optou por modernizar o sistema de rastreamento de sua frota de distribuição, através do uso de software comercialmente disponível para este fim.

Assim, além de poder ter acesso a novas informações e métricas acerca do setor de logística, a gestão local pode aprimorar os métodos de gerenciamento da sua frota e seus respectivos resultados. Logo, para efeito de discussão, serão comparados os dois cenários em que a empresa vivenciou: antes e depois da aplicação do software.

\subsection{Cenário inicial: antes da implantação do software}

A distribuição local é feita por 14 veículos, cada um com dois colaboradores (nas categorias motorista e ajudante). A distribuição dos produtos vendidos abrange a cidade de Petrolina e ainda, em um raio de aproximadamente $80 \mathrm{~km}$, mais algumas vilas no Estado de Pernambuco.

Alguns indicadores são medidos pela gestão do setor, que os gerencia de forma não muito efetiva, por meio de dados informados pelos motoristas e funcionários do depósito da empresa. Assim, número de devoluções, quebras de produtos em movimentação, consumo médio de combustível por rota e outros mais são informados periodicamente.

No setor de logística da empresa, há oito funcionários na área administrativa, dentre eles dois são responsáveis pela verificação e acompanhamento das entregas junto as equipes de entrega. Porém, devido a precariedade da administração, a empresa pôde notar que havia muita divergência entre informações, por isso resolveu implantar o software que ajuda a registrar o que acontece com a operação de distribuição minuto a minuto. 


\subsection{Cenário posterior: depois da implantação do software}

A instalação do programa designado para execução do software foi realizada pela empresa responsável pelo mesmo, durante uma semana no mês de março de 2018 , onde os computadores do setor foram atualizados e modernizados para lidar com as informações e estarem aptos para funcionamento pleno.

O programa instalado é capaz de fornecer várias informações relevantes, como otimização de rotas, sequenciamento de entregas, cadastro digital de clientes, consumo médio de combustível por veículo, registro de velocidade, número de paradas feitas em rota, localização dos carros atualizada instantaneamente, ocupação dos veículos, tempo utilizado para entrega e outras mais.

A equipe local passou por três dias consecutivos de treinamento acerca do software, onde dúvidas puderam ser sanadas além de ensaios e validação que foram feitos também. Após três meses em funcionamento, os funcionários do setor perceberam a diferença nos resultados obtidos por equipe (veículo) e no conjunto. Para efeito de discussão, alguns índices foram postos em comparação para visualização das melhorias.

Tratando-se do consumo médio de combustível (L/Km), observou-se significativa redução em comparação por mês, entre primeiro trimestre de 2018 (janeiro a março) e segundo trimestre (abril a junho), conforme Tabela 1.

Tabela 1 - Consumo médio de combustível por equipe

\begin{tabular}{ccccc}
\hline Equipe & Primeiro trimestre & $\begin{array}{c}\text { Segundo } \\
\text { trimestre }\end{array}$ & $\begin{array}{c}\text { Variação } \\
\text { L/Km }\end{array}$ & $\begin{array}{c}\text { Variação } \\
(\%)\end{array}$ \\
\hline Equipe 1 & 88,5 & 84,1 & 4,4 & $-4,97 \%$ \\
Equipe 2 & 90,1 & 82,9 & 7,2 & $-7,99 \%$ \\
Equipe 3 & 78,4 & 72,7 & 5,7 & $-7,27 \%$ \\
Equipe 4 & 82,2 & 80,1 & 2,1 & $-2,55 \%$ \\
Equipe 5 & 91,4 & 85,6 & 5,8 & $-6,35 \%$ \\
Equipe 6 & 79,9 & 75,5 & 4,4 & $-5,51 \%$ \\
Equipe 7 & 83,8 & 78,4 & 5,4 & $-6,44 \%$ \\
Equipe 8 & 83,6 & 80,3 & 3,3 & $-3,95 \%$ \\
Equipe 9 & 75,5 & 70,9 & 4,6 & $-6,09 \%$ \\
Equipe 10 & 80,0 & 74,5 & 5,5 & $-6,88 \%$ \\
Equipe 11 & 93,2 & 91,7 & 1,5 & $-1,61 \%$ \\
Equipe 12 & 70,6 & 66,8 & 3,8 & $-5,38 \%$ \\
Equipe 13 & 77,9 & 72,8 & 5,1 & $-6,55 \%$ \\
Equipe 14 & 88,4 & 85,0 & 3,4 & $-3,85 \%$ \\
\hline Total & 1163,5 & 1101,3 & 62,2 & $-75,38 \%$ \\
\hline
\end{tabular}

Fonte: Dados da empresa (2018).

De acordo com a gestão do setor, os resultados positivos foram obtidos devido a nova configuração de rotas feitas pelo programa de computador. Agora, as equipes seguem a rota definida de acordo com 0 caminho mais curto, reduzindo assim o consumo de litros de combustível por quilômetro rodado. Em termos de custos, pôde-se notar ainda redução relevante nas despesas com esse tipo de insumo, conforme Tabela 2. 
Tabela 2 - Despesa com combustível

\begin{tabular}{cccc}
\hline & Primeiro trimestre & $\begin{array}{c}\text { Segundo } \\
\text { trimestre }\end{array}$ & $\begin{array}{c}\text { Variação } \\
(\mathrm{R} \$)\end{array}$ \\
\hline Tipo I & $\mathrm{R} \$ 17.541,05$ & $\mathrm{R} \$ 11.003,74$ & $-\mathrm{R} \$ 6.537,31$ \\
Tipo II & $\mathrm{R} \$ 21.366,57$ & $\mathrm{R} \$ 12.163,00$ & $-\mathrm{R} \$ 9.203,57$ \\
\hline Total & $\mathrm{R} \$ 38.907,62$ & $\mathrm{R} \$ 23.166,74$ & $\mathrm{R} \$ 15.740,88$ \\
\hline
\end{tabular}

Fonte: Dados da empresa (2018).

Conforme consta na Tabela 2, houve, de fato, redução expressiva em investimento com combustível. Vale a pena salientar, que os valores não seguem automaticamente a redução composta pela Tabela 1 devido a variação do preço dos combustíveis, principalmente do tipo II (gasolina) ser maior em comparação com o do tipo I (óleo diesel).

Ademais, com o apoio do software, o monitoramento do número de devoluções por equipe também foi minimizado. Antes, os registos mensais eram acima da meta estabelecida de $4,5 \%$ da quantidade de caixas ao dia. Tal evolução pode ser notada na Gráfico 1.

Gráfico 1 - Índice de devolução em \% mensal

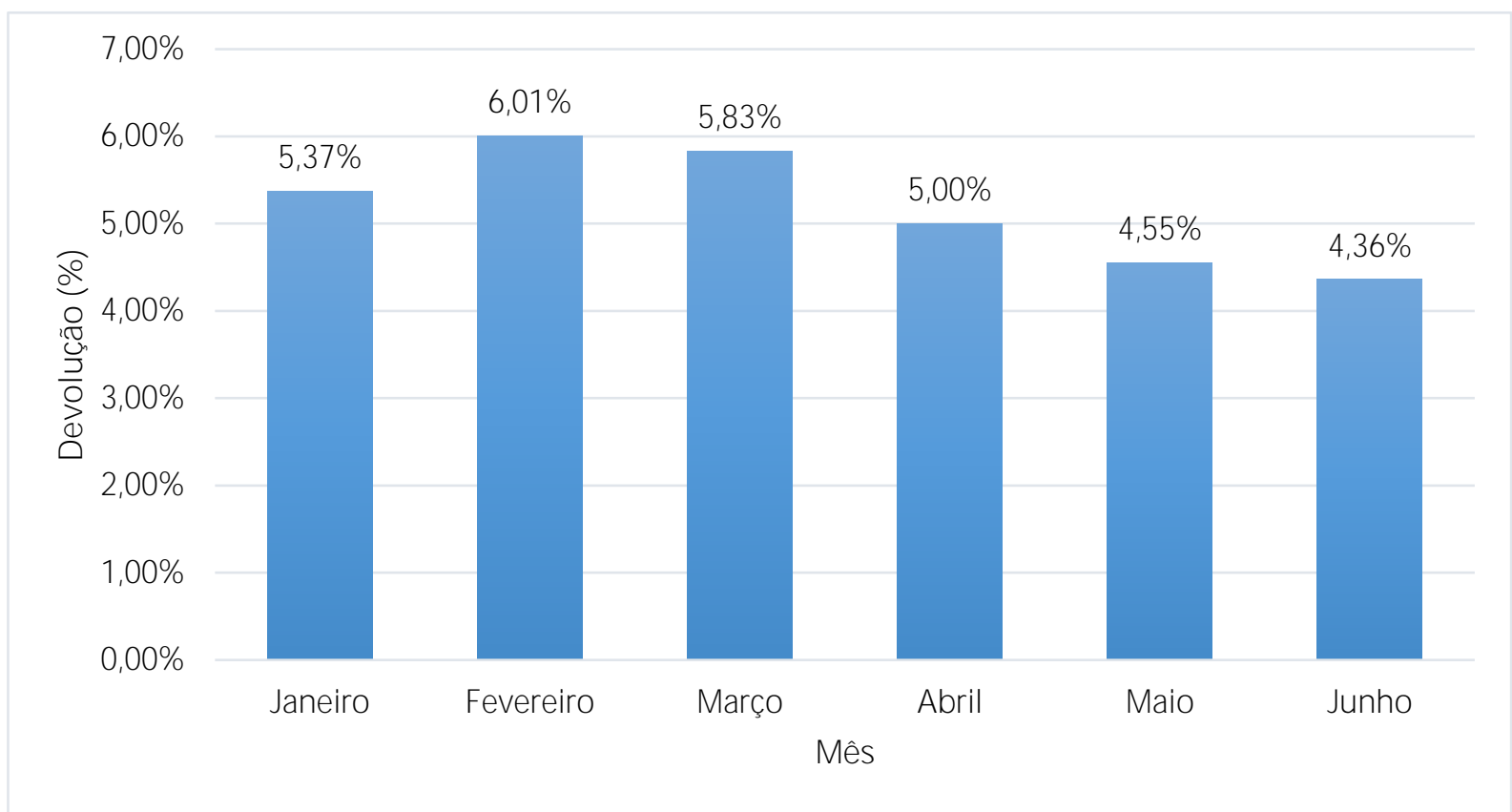

Fonte: Dados da empresa (2018).

A minimização de devoluções registrada pelo setor no primeiro semestre do ano deu-se devido a função do programa que indica a ocorrência de devolução em momento real, dando chance aos colaboradores internos do setor na resolução destas. Geralmente, a comunicação entre setor e funcionários em rota é feita por ligações entre aparelhos de telefone corporativos, mas o programa ainda possui a funcionalidade de enviar mensagens de texto direto aos motoristas.

Outro benefício notado após a instalação e uso do programa de computador é em relação ao tempo médio utilizado nas entregas por equipe. Tal verificação foi percebida através do tempo dispendido pelos colaboradores em uma jornada diária de trabalho. 0 Gráfico 2 mostra tal comportamento. 
Gráfico 2 - Tempo de jornada de trabalho média

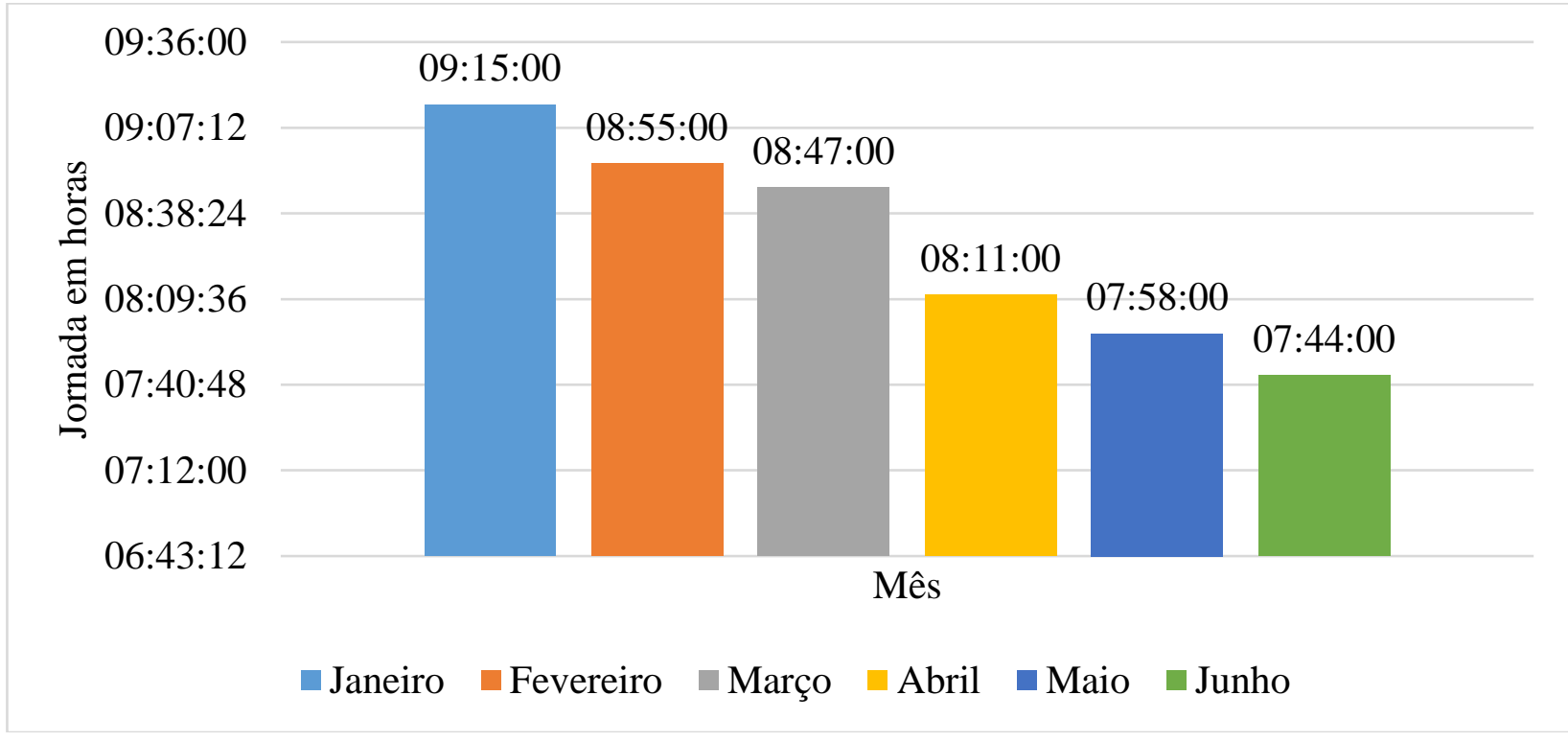

Fonte: Dados da empresa (2018).

Com o acompanhamento das equipes em momento real via software, além das rotas otimizadas, a consequência no tempo médio das equipes de rua disposto no trabalho foi imediata. Assim, com um tempo dentro do aceitável na jornada de trabalho, abaixo de 08h00min por dia, os custos com horas extra dos funcionários foi alcançado, minimizando-se significativamente, conforme Tabela 3.

Tabela 3 - Investimento em hora extra

\begin{tabular}{cccc} 
& Primeiro trimestre & $\begin{array}{c}\text { Segundo } \\
\text { trimestre }\end{array}$ & $\begin{array}{c}\text { Variação } \\
(\mathrm{R} \$)\end{array}$ \\
\hline Hora extra & $\mathrm{R} \$ 3.991,05$ & $\mathrm{R} \$ 2.001,17$ & $\mathrm{R} \$ 1.989,88$ \\
\hline & Fonte: Dados da empresa (2018). &
\end{tabular}

O valor estimado de investimento em hora extra representa grande economia para o setor, uma vez que os casos de necessidade especial de ultrapassar a jornada de trabal ho de 08h00min se dá, a partir de agora, apenas em consideração a razões plausíveis, evitando-se assim ultrapassar esse tempo.

\subsection{Da percepção da equipe de trabalho}

Dos 28 (vinte e oito) colaboradores que trabalham em rota nos 14 (quatorze) veículos da empresa, todos foram entrevistados sobre a percepção em relação a utilização do programa computacional. Contudo, destes, 22 (vinte e dois) estavam aptos a responder, tendo em vista que o restante, 06 (seis), não estavam empregados na organização antes da implementação do software. As respostas para este grupo são exibidas no gráfico 3. 


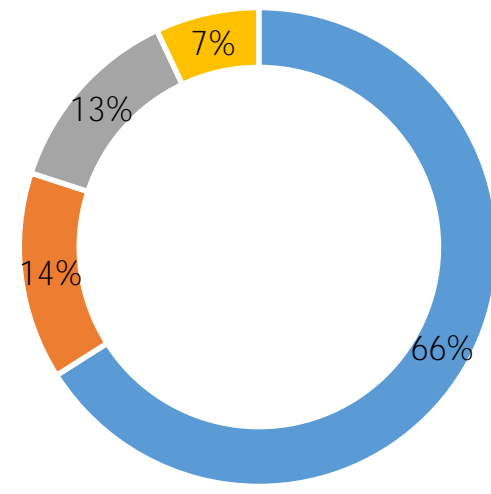

- Grau 1 - Grau 2 - Grau 3 -Grau 4

Fonte: Dados da pesquisa (2018).

Dos funcionários que se submeteram ao questionamento, foi-se posto em questão qual a percepção que eles possuíam em relação ao uso do software no cotidiano do setor. Para isto, foi-se estabelecido quatro opções de resposta, representadas por grau de melhoria. Portanto, os colaboradores tinham a opção de responder:

- Grau 1: Perceberam real mudança nas operações do setor;

- Grau 2: Notaram mudança razoável nas operações do setor;

- Grau 3: Pouca alteração no modo de operar;

- Grau 4: Nenhuma modificação na qualidade das operações e/ou não estou seguro ao opinar.

Além destes, os 08 (oito) integrantes do setor, que trabalham internamente na administração, também foram submetidos aos quesitos acima. Todos deste grupo estavam aptos a responderem e o resultado é visto no Gráfico 4.

Gráfico 4 - Percepção do grupo 2 acerca do software

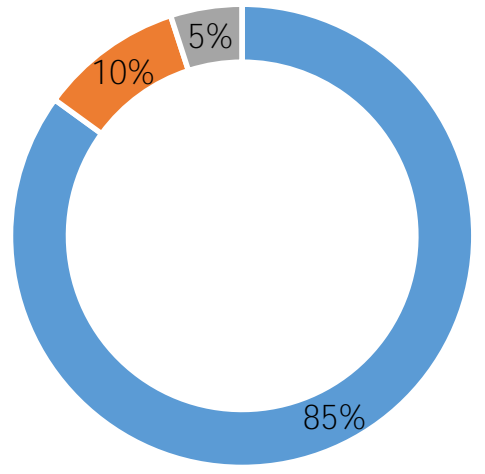

- Grau 1 - Grau 2 - Grau 3 -Grau 4

Fonte: Dados da pesquisa (2018). 
Como observa-se no Gráfico 4, nenhum funcionário assinalou o grau 4. É notório que o programa cumpriu com seu objetivo de melhorar as operações do setor logístico da empresa, trazendo uma abordagem mais realista do que acontece no cotidiano do departamento, facilitando ainda a tomada de decisão por parte da gestão local.

\subsection{Recomendações}

Apesar da ampla observação feita no ambiente empresarial, pode-se perceber que a tecnologia isolada não é capaz de trazer melho rias, isto é, o resultado não é automático. 0 comprometimento das pessoas para com as situações deve ser de total responsabilidade em interpretar da melhor forma as informações dispostas pelo software.

Assim, a gestão deve estar atenta constantemente com a sinergia entre aparato tecnológico e suas vantagens e a participação da equipe de trabalho no compromisso de tornar os resultados positivos sustentáveis. Como ações a serem recomendadas, citam-se:

- Treinamento periódico da equipe local;

- Revisão dos parâmetros e/ou indicadores;

- Retroalimentar o sistema de acordo com novas atualizações disponíveis;

- Executar pesquisa de satisfação com os clientes para averiguar o atendimento às expectativas dos mesmos;

- Envolver os colaboradores com o objetivo do setor, garantindo melhoria contínua nos resultados.

A partir das ações estabelecidas, como recomendação, espera-se que os resultados alcançad os nesses primeiros meses do ano permaneçam estáveis e/ou melhorem continuamente.

\section{CONSIDERAÇÕES FINAIS}

É explícita a importância das tecnologias associadas ao gerenciamento de setores empresariais. Independente do ramo, pode-se obter vantagem competitiva com a utilização de aparato tecnológico e promover a qualidade das operações. 0 presente trabalho mostrou que, quando integradas, as informações podem ser melhor utilizadas na tomada de decisão, auxiliando os gestores.

No presente estudo, pôde-se notar diversos benefícios obtidos através da utilização de um software comercial, destinado ao gerenciamento integrado de informações, em um setor de logística. 0 ganho de eficiência foi comprovado pela gestão local, onde a otimização das operações contribuiu para ganhos efetivos para a empresa. A partir do programa computacional, o setor tornou-se mais ágil, respondendo com maior segurança às demandas diante das situações do cotidiano.

Das melhorias observadas, pode-se chamar atenção para a redução expressiva em investimento com combustível (economizando $\mathrm{R} \$ 15.740,88$ com esse item), além do monitoramento do número de devoluções por equipe que também foi minimizado. Outro benefício notado - após a instalação e uso do programa de computador - é em relação ao tempo médio utilizado nas entregas por equipe.

Tal verificação foi percebida através do tempo dispendido pelos colaboradores em uma jornada diária de trabalho. Com o acompanhamento das equipes em momento real via software, além das rotas otimizadas, a consequência no tempo médio das equipes de rua disposto no trabalho foi imediata, com uma economia de $\mathrm{R} \$ 1.989,88$ no pagamento de horas extra aos funcionários.

Portanto, ficou evidenciado, por meio deste estudo, que a tecnologia pode favorecer a boa gestão seto rial. 0 programa cumpriu com seu objetivo de melhorar as operações do setor, trazendo uma abordagem mais realista do que acontece nas atividades do departamento, facilitando ainda a tomada de decisão por parte da gestão local. 


\section{REFERÊNCIAS}

ANDRADE, R. O. B.; AMBONI, N. Teoria geral da administração: das origens às perspectivas contemporâneas. São Paulo: M. Books do Brasil Editora Ltda, 2007.

APPOLINÁRIO, F. Dicionário de metodologia científica: um guia para a produção do conhecimento científico. São Paulo: Atlas, 2004.

ARAÚJ O, L. C. G. Teoria geral da administração: aplicação e resultados nas empresas brasileiras. São Paulo: Atlas, 2004.

BALLOU, R. H. The Evolution and Future of Logistics and Supply Chain Management. European Business Review, v. 19, n. 4, p. 332-348, 2007.

BARROS, A. J. S.; LEHFELD, N. A. S. Fundamentos de metodologia: um guia para a iniciação científica. 2. ed. São Paulo: Makron Books, 2000.

BEŠLIĆ, I., BEŠLIĆ, D. Measuring performance in a production company as a source of competitive advantage, Economics of Agriculture, v. 57, n. 2, p. 313-326. 2010.

BONAZZI, F. L. Z.; ZILBER, M. A. Innovation and business model: a case study about integration of Innovation Funnel and Business Model Canvas. Revista Brasileira de Gestão de Negócios, São Paulo, v. 16, n. 53, p. 616-637, 2014.

BOWERSOX, D. J.; CLOSS, D. J . Logística empresarial: o processo de integração da cadeia de suprimentos. São Paulo: Atlas, 2001.

BOWERSOX, D. J.; CLOSS, D. J . Logística empresarial: o processo de integração da cadeia de suprimentos. São Paulo/SP. Editora Atlas S.A. 2011

BOWERSOX, D. J .; CLOSS, D. . .; COOPER, M. B. Gestão da cadeia de suprimentos e logística. Rio de Janeiro: Elsevier, 2007.

CERTO, S. C. Administração moderna. 9. ed. São Paulo: Prentice Hall, 2003.

CHANKOV, S. M.; BECKER, T.; WINDT, K. Towards definition of synchronization in logistics systems, Procedia CIRP, v. 17, p. 594-599. 2014.

CHENG, J. H. Inter-organizational relationships and information sharing in supply chains. International Journal of Information Management, v. 31, p. 374-384, 2011.

CHRISTOPHER, M. Logistics \& supply chain management. London: UK, 1992.

CRUZ, T. Sistemas, organizações e métodos: estudo integrado das tecnologias de informação. 3 ed. São Paulo: Atlas, 2007.

DAFT, R. L. Administração. São Paulo: Pioneira Thomson Learning, 2005.

ELMARAGHY, H. et al. Product variety management. CIRP Ann. - Manuf. Technol. v. 62, p. 629-652, 2013.

ESCHENBÄCHER, J.; SEIFERT, M.; THOBEN, K-D. Improving distributed innovation processes in virtual organisations through the evaluation of collaboration intensities. Production Planning \& Control: The Management of Operations, London, v. 22, n. 5-6, p. 473-487, 2011. 
GHOUMRASSI, A.; TIGU, G. The impact of the logistics management in customer satisfaction. In: INTERNATIONAL CONFERENCE ON BUSINESS EXCELLENCE (ICBE), 19., 2017, Paris. Proceedings of ICBE: IRC, 2017. p. 292-301.

GIL, A. C. Como elaborar projetos de pesquisa. 4. ed. São Paulo: Atlas, 2009.

GOVINDARAJA M.; NATARAJ AN S. Principles of management. New Delhi: Published by Prentice Hall Limited, 2005.

GUPTA, U.; RAMESH, A. Analyzing the barriers of healthcare supply chain in India: the contribution and interaction of factors. Procedia- Social and Behavioural Sciences, v. 189, p. 217- 228, 2015.

MUNIZJUNIOR, J. Administração da produção. Curitiba: lesde Brasil. 2012.

KHERBACH, O.; MOCAN, M. L. The Importance of Logistics and Supply Chain Management in the Enhancement of Romanian SMEs, Procedia - Social and Behavioral Sciences, v. 221, p. 405-413, 2016.

KORECKÝ, M. Risk management in logistics. In: CARPATHIAN LOGISTICS CONGRESS (CLC), 2., 2012, Jeseník. Proceedings of CLC. Jeseník, Czech Republic: EU, 2012. p. 1-6.

LAKATOS P. NÉMETH G. Logistics performance assessment. In: MANAGEMENT SCIENCE TODAY INTERNATIONAL SCIENTIFIC CONFERENCE, 1., 2013, Hungary. Proceedings of the "Scientific Management". Hungary : University of Szeged, 2013. p. 277-283.

LAURINDO, F. J . B. Tecnologia da informação: planejamento e gestão de estratégias. São Paulo: Editora Atlas, 2008.

$\mathrm{LI}, \mathrm{X}$. Operations management of logistics and supply chain: issues and directions. Discrete Dynamics in Nature and Society, p. 1-7, 2014.

MAŠIĆ, B.; DŽUNIĆ, M.; NEŠIĆ, S. Savremena teorija menadžmenta - škole i novi pristupi. Beograd: Data status. 2014.

MAXIMIANO, A. C. A. Introdução à administração. São Paulo: Atlas, 2000.

MELVILLE, N. I. S. Innovation for environmental sustainability. MIS Quarterly, v. 34, n. 1, p. 1-21, 2010.

MOURA, A. R. Sistemas e técnicas de movimentação e armazenagem de materiais. São Paulo/SP: ed.IMAM, 2010.

MÜNCHHAUSEN, V. S.; HÄRING, A. M.; KNICKEL, K. The concept of the business logic for the management of values-based food businesses and chains. [2016]. Disponível em: http://orgprints.org/30694/1/Task2\%20Report_version3.pdf . Acesso em: 10 set. 2018.

NOVAES, A. G. Logística e gerenciamento da cadeia de distribuição: estratégia, operação e avaliação. Rio de Janeiro: Campus, 2001.

OSIPOV, G. V.; KURTHS, J ; ZHOU, C. Synchronization in oscillatory networks. Berlin: Springer, 2007.

POTOCAN, V.; NEDELKO, Z.; MULEJ , M. Influence of Organizational Factors on Management Tools Usage in Slovenian Organizations. Engineering Economics, v. 23, n. 3, p. 291-300, 2012.

QUETTI, C.; PIGNI, F.; CLERICI, A. Factors affecting RFId adoption in a vertical supply chain: the case of the silk industry in Italy. Production Planning \& Control: The Management of Operations, London, v. 23, n. 4, p. 315-331, 2012. 
REEVES, M.; DEIMLER, M. Adaptability: the new competitive advantage. Harvard Business Review, v. 89, n. 78, p. 134-141, 2011.

REZENDE, D. A.; ABREU A. F. Tecnologia da informação aplicada a sistemas de informações empresariais: o papel estratégico da informação e dos sistemas de informação nas empresas. 8. ed. São Paulo: Atlas, 2011.

SANTOS, P. V. S.; ROCHA, M. F. de B. Inovação no processo de desenvolvimento de estratégias competitivas em pequenas e médias empresas. Revista Mundi Engenharia, Tecnologia e Gestão, v. 2, p. 1-48, 2017.

SILVA, E. L.; MENEZES, E. M. Metodologia da pesquisa e elaboração de dissertação. 4. ed. Florianópolis: Laboratório de Ensino a Distância da Universidade Federal de Santa Catarina, 2005.

SO, S.; SUN, H. Supplier integration strategy for lean manufacturing adoption in electronic-enabled supply chains. Supply Chain Management, v. 15, p. 474-487, 2010.

TATARYNOWICZ, A.; SYTCH, M.; GULATI, R. Environmental demands and the emergence of social structure: Technological dynamism and interorganizational network forms. Administrative Science Quarterly, v. 61, n. 1, p. 52-86, 2016.

VAN CREVELD, M. Supplying war. New York, NY: Cambridge University Press, 2004.

VIJ AYASARATHY, L. R. An investigation of moderators of the link between technology use in the supply chain and supply chain performance. Information \& Management, v. 47, p. 364-371, 2010.

YAYLA, A. Y. et al. A hybrid data analytic methodology for 3PL transportation provider evaluation using fuzzy multi-criteria decision making, International J ournal of Production Research, v. 53, n. 20, p. 6097-6113, 2015.

YIN, R. K. Estudo de caso: planejamento e métodos. 3. ed. Porto Alegre: Bookman, 2005. 212 p.

YUCESAN, E. The impact of information technology on supply chain management. In: JUNG, H.; EONG, B.; CHEN, F. F. (org.). Trends in Supply Chain Design and Management: Technologies and Methodologies. London: Springer, 2007. cap. 6. 